\title{
The assessment of prophylactic and therapeutic methods for nail infections in patients with diabetes
}

\author{
Kamila Korpowska1, Marianna Majchrzycka², Zygmunt Adamski² \\ ${ }^{1}$ Podiatry Institute, Great Poland Podiatry Center, Poznan, Poland \\ ${ }^{2}$ Department of Dermatology, Heliodor Święcicki Clinical Hospital of the Poznan University of Medical Sciences, Poznan, Poland \\ Adv Dermatol Allergol 2022; XXXIX (6): 1048-1052 \\ DOI: https://doi.org/10.5114/ada.2022.113585
}

\begin{abstract}
Introduction: Diabetes mellitus is a metabolic disease widespread around the world. It may lead to organ dysfunction, immunodeficiency, vascular complications, and peripheral neuropathy. These factors contribute to susceptibility to fungal and bacterial infections of the nails, which could have serious consequences.

Aim: To evaluate the knowledge about infection prophylaxis, prevalence of pathogens and a change in quality of life among different groups of diabetic patients.

Material and methods: The study was performed using the DLQI questionnaire, interview, physical examination, mycological tests, and a survey containing multiple choice questions. The sample consisted of 120 patients. We revealed the presence of the infection in almost all of the patients, both with type 1 and type 2 diabetes.

Results: A great number of the participants lacked a credible source of information on their disease and consequently had little understanding of possible complications and prophylaxis of the nail infections. An overwhelming majority of the patients experienced pronounced discomfort of the skin and nails and felt extremely embarrassed with their nail appearance.

Conclusions: Further research needs to be conducted to determine the efficacy of different methods of preventing nail infections in diabetic patients. Physicians, aside from implementing appropriate treatment, need to ensure that high-risk patients receive sufficient education on the prophylaxis of nail infections and on proper foot care.

Key words: onychomycosis, nails, diabetes mellitus.
\end{abstract}

\section{Introduction}

Diabetes mellitus is a metabolic disease, which is characterized by an elevated plasma glucose level. Considering its widespread occurrence in human population, it poses a major challenge to public health. The incidence of diabetes is steadily increasing worldwide. It is inter alia the repercussion of an ongoing rapid urbanization, which results in lowering the levels of physical activity and gaining weight. Low socioeconomic status is also a risk factor for diabetes [1].

Untreated diabetes mellitus leads among others to organ dysfunction, immunodeficiency, severe vascular complications, and peripheral neuropathy. Early diagnosis and prevention of typical consequences of the disease are a crucial task of health professionals.

Vascular, immunological and neuropathic complications may lead to the development of various diseases of the skin, nails and the diabetic foot syndrome, which is a relatively frequent cause of limb loss [2].

Nails significantly improve manual abilities of the hands, enabling the precision of grip and the handling of objects. Hard nail plate protects plentiful nerve endings from injuries [3]. Nails also contribute greatly to hands and feet aesthetics, and consequently to patients' general well-being. Their appearance also plays a role in diagnostics, since just like the appearance of the skin, it correlates with various abnormal conditions of the body, such as diseases of skin and connective tissues as well as infections [4].

Any lesion on the nail, when discovered, requires a thorough differential diagnosis, implementing suitable treatment and relapse prevention. The aetiology of a lesion can be infectious or systemic, as for instance in nail psoriasis, often accompanying diabetes mellitus [5].

Address for correspondence: Marianna Majchrzycka MD, Department of Dermatology, Heliodor Święcicki Clinical Hospital of the Poznan University of Medical Sciences, Poznan, Poland, e-mail: mmajchrzycka@ump.edu.pl Received: 16.12.2021, accepted: 1.1.2022. 
Susceptibility to bacterial and fungal infections of the nail is conditioned by numerous factors, such as age, sex, genetic predisposition, preferred footwear type, smoking, attending public swimming pools [6]. Diabetes is also one of the key risk factors in developing onychomycosis, whose versatile clinical presentation needs to be differentiated, among others, with consequences of atherosclerosis of the peripheral arteries and mechanical traumas, unnoticed by the patient due to peripheral neuropathy [7].

The most common type of onychomycosis is distal and lateral subungual onychomycosis, known as DLCO. It involves the nail plate starting from the free edge or lateral nail folds. Nail bed is also infected and becomes pathologically hyperkeratotic. This leads to the elevation of the nail plate, which changes its colour to yellowishbrown and becomes uneven. Diabetics are also susceptible to bacterial infections, such as paronychia or infection caused by Pseudomonas aeruginosa. This process is often preceded by onycholysis and a destruction of the nail unit [8].

\section{Aim}

The aim of this study is to evaluate the level of knowledge among patients with diabetes mellitus about ways of preventing nail infections and about the impact that these infections have on the patients' lives. We also analysed differences between groups of participants - with type 1 and type 2 diabetes, as well as between males and females. The final part of the article contains two case reports, in which we will demonstrate different therapeutic approaches in diabetic patients with nail infections.

\section{Material and methods}

The study was performed using the DLQI questionnaire, interview, physical examination, mycological tests, and a survey containing multiple choice questions. One-hundred and twenty patients with diabetes who attended the podiatry clinic to evaluate changes of the nail plate were enrolled in the study. Half of the respondents suffered from type 1 , and half from type 2 diabetes. Examination was conducted at the Department of Dermatology in Heliodor Święcicki Clinical Hospital in Poznan. Concomitant diseases of the participants had been verified by specialists in their respective fields. The material has been acquired according to modern ethical standards and the study has been approved by the legally appropriate ethical committee.

\section{Results}

\section{Type 2 diabetes}

Among the 60 participants with type 2 diabetes, 72\% had higher education. All of the women were at least
50 years of age and all of the men at least 60 . The duration of their disease exceeded 10 years. The whole group had been under permanent control of a diabetologist. Everyone lived in the city with a population over 500 thousand.

The results of the mycological examination and fungal culture tests revealed infection of the nail plate in $87 \%$ of the patients. Dermatophytes were found to be the most common fungus (62\%), yeast-like and mould fungi were also cultivated from the samples. A high prevalence of bacteria was also discovered (83\%). Onycholysis was found in all of the patients, discoloration of the nail plate and subungual hyperkeratosis in $88 \%$ and $93 \%$ of the sample respectively.

Only slightly more than a half (56\%) of the respondents stated they gained knowledge about their disease from doctors, and $18 \%$ of them considered Internet articles as their main source of information. According to $62 \%$ of the patients (of whom 65\% were men), properly fitting footwear type does not influence the risk of developing the diabetic foot syndrome. Only one female wore high heels on a daily basis. In order to prevent disorders of the skin and nails, $27 \%$ of the participants visited a podiatrist regularly, and $43 \%$ limited their actions to clipping their toenails. Roughly half (53\%) of the sample reported very pronounced ailments of the skin in the week before, taking the form of pruritus, pain, stinging or tingling (the frequencies of the answers are provided in Figure 1). An overwhelming majority (90\%) felt extremely embarrassed with the appearance of their skin and nails, and 50\% recognized a huge negative impact of these conditions on their social life and ways of spending free time.

\section{Type 1 diabetes}

Patients with type 1 diabetes fell within three age groups - 16 people under 18 years old, 41 between 19 and 32 , and 3 people over 33 years of age. Most of them (58\%) were diagnosed with diabetes less than 5 years before, $12 \%$ over 10 , and the rest (30\%) between 6 to 10 years. Individuals with higher education made up $17 \%$ of the group, while $88 \%$ of the participants were professionally active. Similarly to the type 2 diabetics sample, all of them lived in a city with a population of more than 500 thousand.

In mycological examination $80 \%$ of the patients tested positive and, just as in the type 2 sample, dermatophytes turned out to be dominant (58\%). Prevalence of onycholysis reached $95 \%$, subungual hyperkeratosis $85 \%$, and discoloration of the nail plate $-58 \%$. Figure 2 presents the occurrence of fungal infections in the whole sample, with a division into type 1 and type 2 diabetes patients.

Over half of the patients acquired information about diabetes and its complications mainly from the Internet (64\%), 23\% sought advice from their doctors, $8 \%$ from 


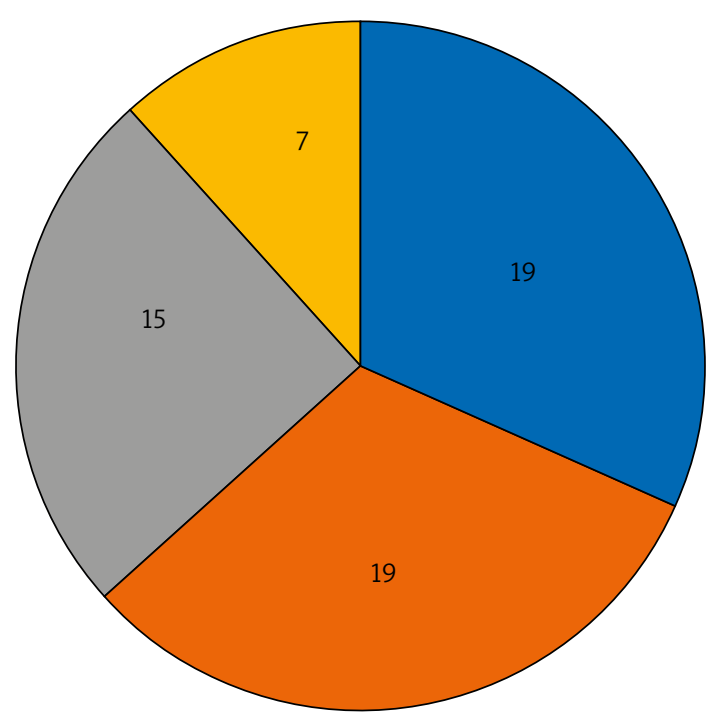

Extremely high $\square$ High $\square$ Moderate $\square$ None

Figure 1. Number of patients with type 1 diabetes and declared level of nail discomfort $(n=60)$

their friends and family, and 5\% educated themselves with books. A majority of the respondents believed there is no connection between the type of footwear and a risk of developing diabetic foot syndrome. One third of the women wore high heels. Twenty-seven percent of the patients visited a podiatrist regularly, $82 \%$ of whom were women. According to the results of the DLQI questionnaire, $63 \%$ of participants experienced pronounced discomfort of the skin and nails in the form of pruritus, pain, stinging or tingling (data provided in Figure 3). Over half of them admitted feeling highly embarrassed with their nail appearance and 62\% acknowledged its negative impact on their social and romantic life.

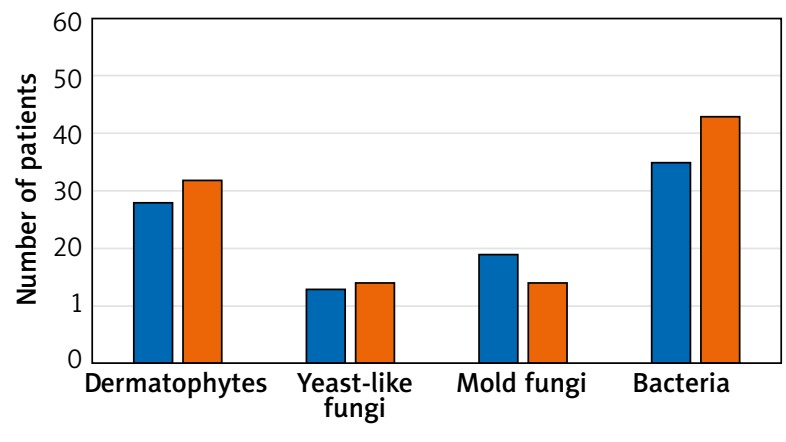

$\square$ Type 1 diabetes $\square$ Type 2 diabetes

Figure 2. Prevalence of pathogens in patients with type 1 and 2 diabetes $(N=120)$

\section{Discussion}

Almost all of the study participants tested positive for onychomycosis. However, the fact that the sample was recruited from volunteers who wanted to rule out the presence of fungal infection needs to be taken into consideration. According to the meta-analysis performed by Gupta et al. in 2014, diabetic patients develop all types of onychomycosis many times more often than healthy individuals [6]. Researchers link this regularity with the coexistence of neuropathy, immunodeficiency, and peripheral vascular disease. The results we obtained did not show a significant difference between the two types of diabetes, or the patients' gender. In the physical examination, onycholysis was found in all of the participants, and was often associated with subungual hyperkeratosis or discoloration. These lesions had been developing over a long period of time, however, they did not alarm the patients sufficiently to see a dermatologist or podiatrist. Moreover, a vast majority of the respondents in both groups found recent skin and nail discomfort greatly disturbing.

While small and demarcated fungal lesions do not pose a real threat to patients' health, untreated onychomycosis might become dangerous, especially for diabetics. Deformation of the nail plate can lead to its ingrowth and subsequently to injuring the lateral nail folds and skin of the fingertip [9]. Neuropathy, peripheral artery disease and immunodeficiency facilitate bacterial superinfection, which might result in cellulitis, osteomyelitis and even sepsis.

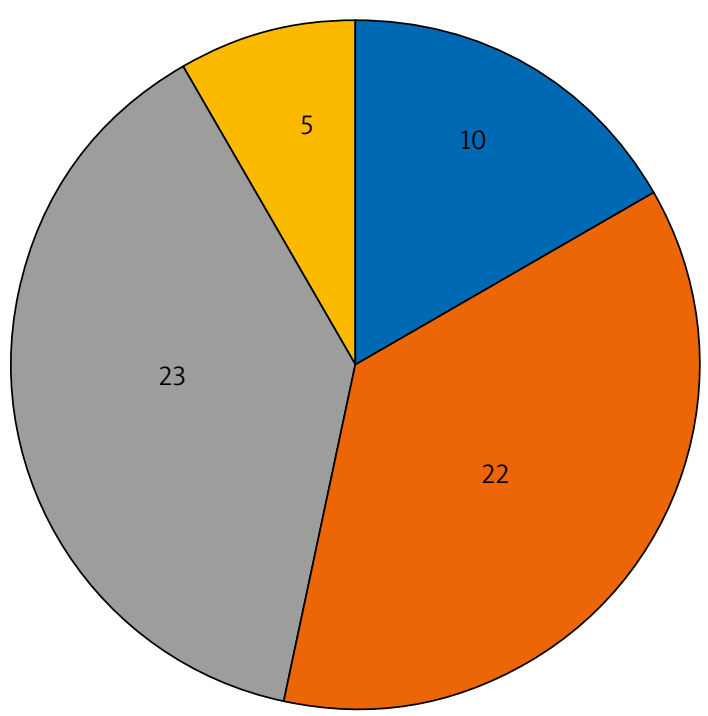

Extremely high $\square$ High $\square$ Moderate $\square$ None

Figure 3. Number of patients with type 2 diabetes and declared level of nail discomfort $(n=60)$ 
Patients' neglect of the symptoms may be the result of a lack of adequate knowledge regarding prophylaxis, coming from a reliable medical source. With regard to the age structure, among the type 1 diabetes sample, younger than that with type 2, the most popular source of information was the Internet. Our questionnaire survey also showed that the respondents were not fully aware of the consequences of wearing poorly-fitting shoes for developing skin infections.

In addition to undeniable risks to physical health, a negative psychological impact of nail plate infection needs to be taken into consideration. A clear majority of the affected felt extremely embarrassed because of the appearance of their nails, which made maintaining and building relationships challenging. This issue should serve as an additional motivation for healthcare professionals of all areas of specialization to ensure that high-risk patients receive sufficient education on the prophylaxis of nail infections and on proper foot care. Basic recommendations should comprise thorough daily selfexamination of the skin and nails, and regular visits to the podiatrist or dermatologist. Patients also need to be aware of the fact that neuropathy often eliminates pain signals arising from developing infection. Pain, however, is a predominant symptom prompting patients to see a doctor.

Treating diabetes and its consequences poses a considerable challenge to numerous branches of medicine. Further research needs to be conducted to determine the efficacy of different methods of preventing nail infections in diabetic patients in order to avoid severe complications, such as the need for amputation of the limb or even death. It is also a physician's task to implement appropriate treatment, stopping the infection from spreading.

\section{Case reports}

In the final part of this work, we present two case reports of the patients of the Dermatology Clinic in the Heliodor Święcicki Clinical Hospital in Poznan, along with a description of the treatment management and the photographs of its effects.
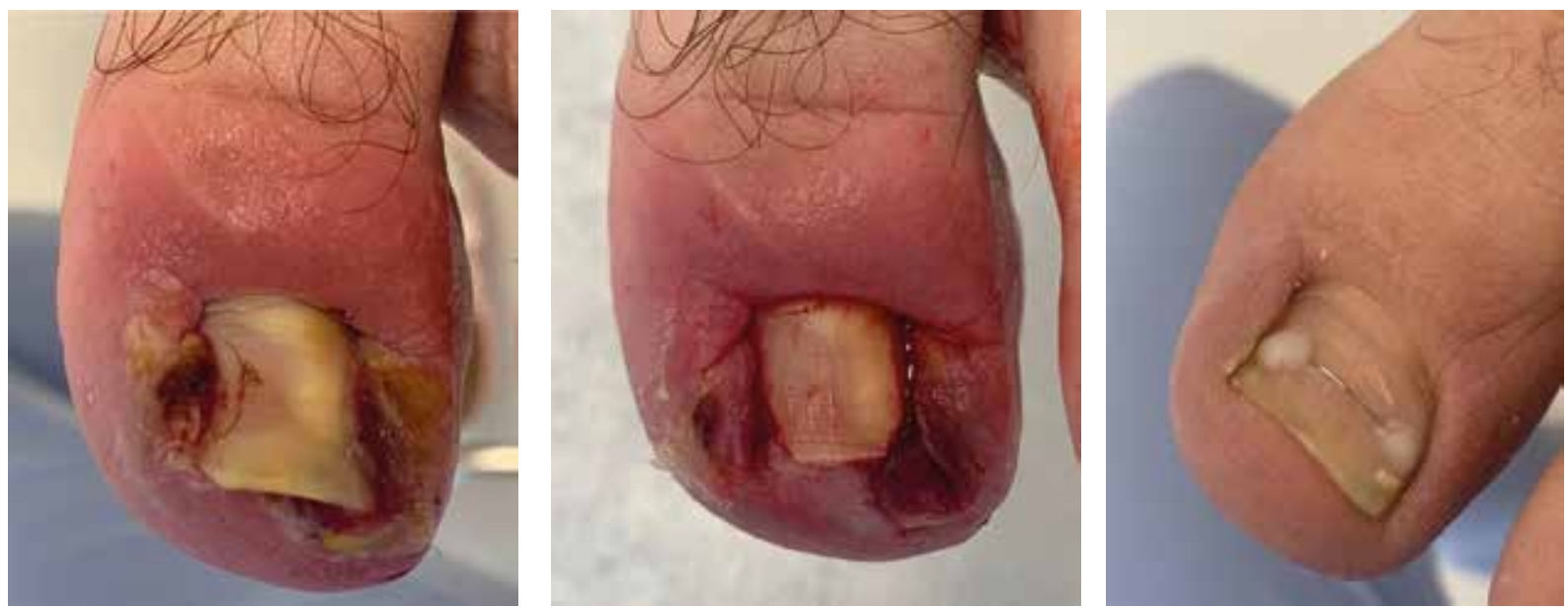

Figure 4. From left to right - patient's toenail on the $1^{\text {st }}, 2^{\text {nd }}$ and $5^{\text {th }}$ visit

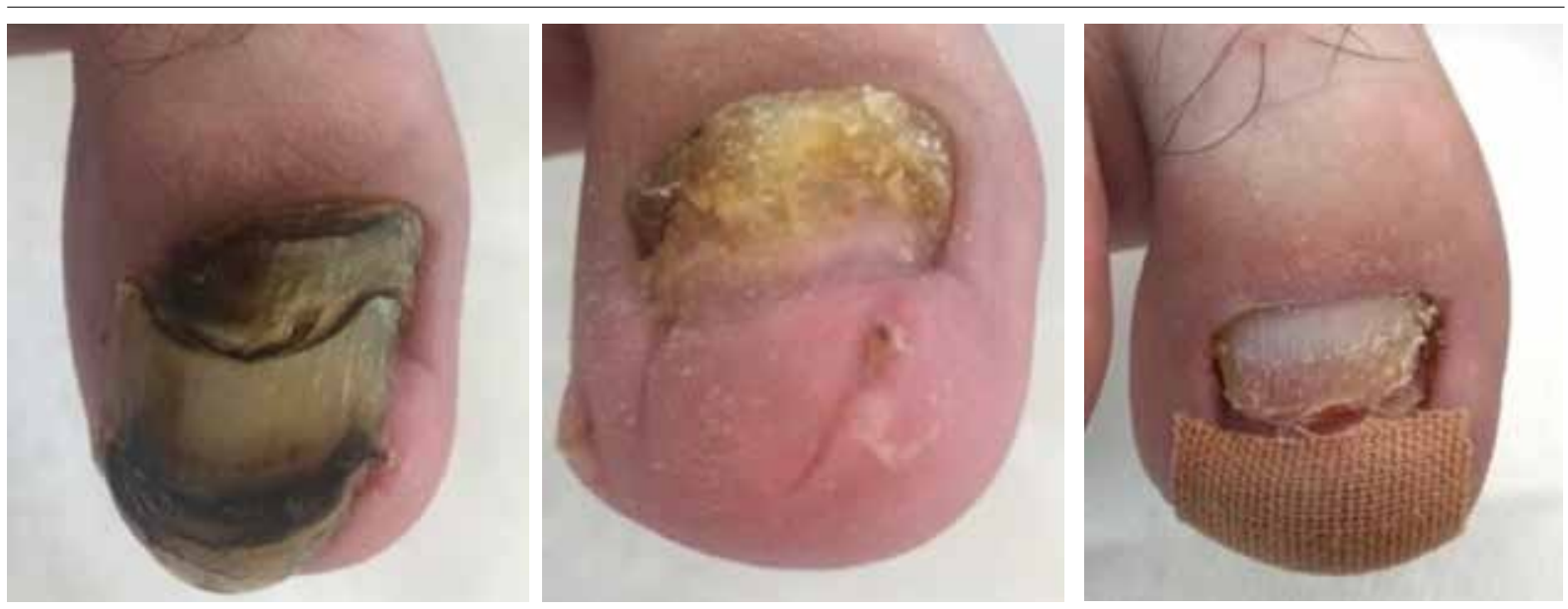

Figure 5. From left to right - patient's toenail on the $1^{\text {st }}, 2^{\text {nd }}$ and $7^{\text {th }}$ (after 7 months) visit 


\section{Case 1}

A 28-year-old male with type 1 diabetes diagnosed 5 years ago sought medical attention in regard to the inflammation, swelling and bleeding of the left foot toenail. Five years before he was subjected to nail avulsion due to a fungal infection diagnosed by a primary care physician and confirmed with mycological tests. The removed nail plate was growing into the skin despite multiple interventions.

The performed tests detected the bacterial infection. Lateral, protruding fragments of the nail plate were removed and a dressing with an antibiotic ointment Betadine (povidone-iodine) was put on. The patient was instructed to use Daktarin (miconazole) cream topically twice a day and additionally the protective liquid $08 \mathrm{OIL}$, containing a mixture of plant oils. Soon after the first visit, the pain diminished. On subsequent visits the antibiotic ointment tamponade was changed. During the fifth visit a titanium toenail brace, which enabled the lift of the nail plate, was applied. Every 3 weeks the patient visited a dermatologist to assess the condition of the toenail brace and the nail itself (Figure 4).

\section{Case 2}

Five years before making an appointment in the Dermatology Clinic, a 42-year-old male with type 2 diabetes and hypertension suffered an injury to the right foot toenail. As a consequence, the nail plate thickened and deformed its shape. A physical examination also showed onycholysis as well as green and black discoloration.

The mycological test revealed the infection with mould fungi Fusarium sp. In accordance with the test results, oral antifungal medication (itraconazole) was introduced. After cleaning and trimming the nail plate on the first visit, the pain ceased. During monthly repeated visits the nail plate was cleaned and examined. The patient was also recommended to use Daktarin (miconazole) cream and 08 OIL topically twice a day (Figure 5).

\section{Conflict of interest}

The authors declare no conflict of interest.

\section{References}

1. Lovic D, Piperidou A, Zografou I, et al. The growing epidemic of diabetes mellitus. Curr Vasc Pharmacol 2020; 18: 104-9.

2. Barnes JA, Eid MA, Creager MA, Goodney PP. Epidemiology and risk of amputation in patients with diabetes and peripheral artery disease. Arterioscler Thromb Vasc Biol 2020; 40: 1808-17.

3. Piraccini BM. Choroby paznokci. Wydawnictwo Czelej, Lublin 2018.

4. Martini MC. Kosmetologia i farmakologia skóry. Wydawnictwo Lekarskie PZWL, Warszawa 2006.

5. Wykłady podologiczne cz. III. Nowicka S (ed.). Wydawnictwo Lekarskie PZWL, Warszawa 2016.
6. Gupta AK, Daigle D, Foley KA. The prevalence of culture-confirmed toenail onychomycosis in at-risk patient populations. J Eur Acad Dermatol Venereol 2015; 29: 1039-44.

7. Aragón-Sánchez J, López-Valverde ME, Víquez-Molina G, et al. Onychomycosis and tinea pedis in the feet of patients with diabetes. Int J Low Extrem Wounds 2021, doi: $10.1177 / 15347346211009409$.

8. Ziaja D, Sznapka M, Kucznik W. Podologia - podręcznik dla studentów medycyny, podologii, kosmetologii. Via Medica, Gdańsk 2020.

9. Rich P, Hare A. Onychomycosis in a special patient population: focus on the diabetic. Int I Dermatol 1999; 38: 17-9. 